\title{
El Pirofosfato de Tiamina Reduce el Daño Celular Inducido por Hipoxia en el Cerebro de Ratas Neonatas
}

\author{
Thiamine Pyrophosphate Reduces Hypoxia-Induced Cellular Damage in Neonatal Rat Brain
}

\author{
Armando Valenzuela Peraza*,; Marco A. Martínez Ávila**; Miguel Ángel Jiménez Bravo-Luna***; \\ Leticia Granados-Rojas*; Julieta G. Mendoza-Torreblanca* \& M. Gerardo Barragán Mejía***
}

\begin{abstract}
VALENZUELA, P. A.; MARTÍNEZ, A. M. A.; JIMÉNEZ, B. M. A.; GRANADOS-ROJAS, L.; MENDOZA-TORREBLANCA, J. G. \& BARRAGÁN, M. M. G. El Pirofosfato de Tiamina reduce el daño celular inducido por hipoxia en el cerebro de ratas neonatas. Int. J. Morphol., 32(2):531-536, 2014.
\end{abstract}

RESUMEN: La encefalopatía por hipoxia es causa de discapacidad y requiere de nuevas estrategias terapéuticas. El pirofosfato de tiamina (PPT) es un cofactor esencial de enzimas fundamentales en el metabolismo de la glucosa, cuya disminución puede conducir a la falla en la síntesis de ATP y a la muerte celular. El objetivo de este estudio fue determinar si la administración de PPT, puede reducir el daño celular en un modelo de hipoxia neonatal en ratas. Animales de 11 días de edad fueron tratados con PPT (130 mg/kg) en dosis única o solución salina, una hora antes del protocolo de hipoxia o al término de ésta. Los cerebros fueron colectados para la evaluación del daño celular. Además, se tomaron muestras sanguíneas para evaluar los indicadores gasométricos de presión de dióxido de carbono $\left(\mathrm{PaCO}_{2}\right)$ y de oxígeno $\left(\mathrm{PaO}_{2}\right)$ en sangre arterial y pH. Los resultados muestran que la administración de PPT previa a la inducción de hipoxia, reduce el daño celular y restablece los indicadores gasométricos. Estos datos indican que el uso de PPT reduce el daño inducido por la hipoxia en animales neonatos.

PALABRAS CLAVE: Pirofosfato de tiamina; Hipoxia; Daño celular; Gasometría hemática.

\section{INTRODUCCIÓN}

Con una incidencia de entre 1 y 6 por 1000 recién nacidos vivos (Ferriero, 2004) la hipoxia es causa importante de morbimortalidad cuando ocurre en la etapa neonatal. Las secuelas más importantes asociadas son: parálisis cerebral, discapacidad cognoscitiva y epilepsia (DelivoriaPapadopoulos \& Mishra, 1998).

$\mathrm{Al}$ ocurrir un evento severo, la reserva energética del tejido se agota. Ante la falta de energía necesaria para restablecer el funcionamiento normal, la célula entra en una fase de parálisis metabólica que la puede llevar a la muerte (Martin et al., 2003). Aunado al evento hipóxico, se desencadenan una serie de procesos: acidosis láctica, incremento en los niveles de glutamato, generación de especies reactivas de oxígeno y estrés oxidante, acumulación de calcio intracelular y activación de la respuesta inflamatoria inmune, los cuales continúan generando daño al cerebro por muchas horas después de que el aporte normal de oxígeno ha sido restablecido (Delivoria-Papadopoulos \& Mishra).
Varias alternativas terapéuticas han sido utilizadas para el tratamiento de las lesiones secundarias a eventos hipóxicos; entre ellas: hipotermia, administración de atrapadores de radicales libres, bloqueadores de canales de calcio y moduladores de los canales K(+)-ATP (Sameshima \& Ikenoue, 2013); algunas han sido incorporadas a la práctica clínica con relativo éxito. Sin embargo, aún no ha sido posible desarrollar un tratamiento dirigido a prevenir el daño celular generado por un evento hipóxico. Tomando en consideración que el daño es progresivo y depende en gran medida del aporte energético a la célula, una de las alternativas podría ser la administración de moléculas que actúen en las vías enzimáticas relacionadas con la producción de energía con el objeto de reducir la magnitud del daño.

La tiamina es una vitamina que ejerce su acción metabólica principalmente a través del pirofosfato de tiamina (PPT), el cual es un cofactor indispensable en la activación de la piruvato deshidrogenasa (PDH), la alfa-ceto glutarato

* Laboratorio de Neuroquímica, Instituto Nacional de Pediatría, México D.F., México.

** Nutrición experimental, Instituto Nacional de Pediatría, México D.F., México.

*** Laboratorio de Cirugía Experimental, Instituto Nacional de Pediatría, México D.F., México.

Dirección de Investigación, Instituto Nacional de Pediatría, Secretaría de Salud, México. Esta investigación fue patrocinada con los fondos federales del Instituto Nacional de Pediatría (protocolo aprobado con número 05/061). 
deshidrogenasa ( $\alpha-\mathrm{CGDH})$, la alfa-ceto ácido deshidrogenasa y la trancetolasa; enzimas involucradas en los procesos de generación de energía a través del ciclo de Krebs o de las pentosas (Martin, et al.). Estudios previos, han demostrado que el encéfalo es muy sensible a la disminución de la concentración de tiamina y que esto puede producir alteraciones del aporte de energía proveniente del metabolismo oxidativo de los nutrientes (Hass, 1988).

Anteriormente, se ha reportado la capacidad de la tiamina para proteger a cardiomiocitos cultivados de ratas neonatales en contra del estrés hipóxico (Shin et al., 2004). También se ha observado que la administración de PPT produce un efecto anti-hipóxico atribuido al incremento en la utilización de alfa-cetoglutarato en el tejido nervioso a través de la activación de las vías de la $\alpha$-CGDH y la Gaba transaminasa (Rozanov et al., 1990). Sin embargo, se desconoce el efecto de la administración in vivo de PPT, sobre el tejido nervioso, ante la exposición a un evento hipóxico. Por ello, el objetivo del presente estudio fue investigar el potencial protector del PPT utilizando un modelo de daño neuronal inducido por hipoxia neonatal.

\section{MATERIAL Y MÉTODO}

Se utilizaron ratas macho Wistar de 11 días de edad (P11) ya que de acuerdo con Vannucci et al. (1999), el desarrollo del cerebro a esta edad es histológicamente similar al de los infantes humanos cercanos al nacimiento. El procedimiento para todos los animales fue aprobado por el comité de ética institucional.

Los animales para experimentación fueron obtenidos de ratas con fecha de gestación similar, las cuales fueron alojadas en cajas individuales con libre acceso al agua y al alimento. Las crías permanecieron con sus madres hasta el inicio de las maniobras experimentales y fueron asignadas de manera aleatoria a los siguientes grupos ( $\mathrm{n}=10$ crías): 1. Control sin manipulación, 2. Con administración de PPT $(130 \mathrm{mg} / \mathrm{kg} / \mathrm{ip})$ una hora antes de ser sometidos a hipoxia (H+PPTpre), 3. Con administración de PPT (130 mg/kg/ip) inmediatamente después de ser sometidos a hipoxia (H+PPTpost), 4. Con administración de Solución Salina una hora antes de ser sometidos a hipoxia (H+SSpre), 5. Con administración de Solución Salina inmediatamente después de ser sometidos a hipoxia (H+SSpost)

Modelo de hipoxia. El modelo consiste en la exposición de los animales a 8\% de oxígeno (Rice et al., 1981) balanceado con nitrógeno, a una temperatura de $35^{\circ} \mathrm{C}$ y humedad relativa de $60-70 \%$, por periodos de 120 min con 24 h de recuperación.
Para la exposición se utilizó una cámara de acrílico, con temperatura controlada, con atmósfera homogénea y control de los niveles de oxígeno por medio de un oxímetro (MiniOX III, Medical Products PITTSBURGH, PA, 15230). Al momento de introducir los animales a la cámara, la concentración de oxigeno tenía una saturación del $21 \%$, posteriormente se administró una mezcla de gases que contenía $10 \%$ de dióxido de carbono $\left(\mathrm{CO}_{2}\right)$ y $90 \%$ de nitrógeno (Vannucci et al., 2001). En el otro extremo la válvula de salida y por medio de un oxímetro (el cual tiene un sensor de oxígeno en la cámara de gases), se monitoreó la concentración de oxígeno a fin de mantener la concentración al 8\%. Después de 120 min de exposición a la mezcla de gases, los animales fueron retirados y se dejaron recuperar durante 15 min bajo condiciones ambientales. Los animales fueron regresados con sus madres por $24 \mathrm{~h}$, y al término de este tiempo fueron sacrificados para la evaluación histológica y gasométrica.

Análisis histológico. Veinticuatro horas posteriores al tratamiento, todos los animales fueron anestesiados con pentobarbital (70 mg/kg/ip), perfundidos por vía intracardíaca, con solución salina y posteriormente con paraformaldehído al $4 \%$ en buffer de fosfatos $0,1 \mathrm{M}, \mathrm{pH} 7,4$. Los cerebros fueron removidos rápidamente y colocados en la misma solución fijadora por $24 \mathrm{~h}$. El encéfalo se dividió en tres secciones coronales, los bloques de tejido se incluyeron en parafina y se obtuvieron cortes coronales de $5 \mu \mathrm{m}$ cada $250 \mu \mathrm{m}$. Los cortes fueron teñidos con fuccina ácida/tionina (Gunn et al., 1997) para la valoración del daño celular.

Dos observadores ciegos a los procedimientos experimentales, clasificaron la presencia y grado de daño en los cerebros de cada animal. El criterio para la semicuantificación de células dañadas por campo fue de acuerdo a cuatro categorías. $-=$ ausencia de daño $0 ; *$ * escaso $1-10$; $* *=$ moderado $11-30 ; * * *=$ abundante 31 en adelante. Las células que presentaban una gran afinidad al colorante (picnóticas) se tomaron cómo células dañadas (Langmeier et al., 2006).

Gasometría hemática. Para comprobar que se generó el evento hipóxico, se cuantificaron los niveles de $\mathrm{pH}, \mathrm{PaCO}_{2}$ y $\mathrm{PaO}_{2}$ (Vanucci et al., 2001). Veinticuatro horas después del evento hipóxico se tomaron muestras sanguíneas de la aorta a nivel abdominal con un microtomador de muestras (MultiCaps-S, Siemens Healthcare Diagnostics Inc., Tarrytown, NY 10591.5097 USA), de $220 \mu \mathrm{l}$ con 6UI de heparina de sodio y 9 UI de heparina de Litio por $100 \mu \mathrm{l}$. El análisis se llevó a cabo con un analizador de gases sanguíneos (Rapidlab 1200, Siemens Healthcare Diagnostics Inc., Tarrytown, NY 10591.5097 USA). Se consideraron como valores normales 7,35-7,45 para él pH; 35-45 mmHg para él $\mathrm{PaCO}_{2}$ y de 80$100 \mathrm{mmHg}$ para él $\mathrm{PaO}_{2}$ (Zehendner et al. 2013). 
Estadística. Los datos se presentan como promedio \pm error estándar (EE) en gráficos y tablas. Para la comparación de los valores de los diferentes indicadores entre los grupos experimentales, se utilizó la prueba de análisis de varianza (ANOVA), previa comprobación de la homogeneidad de las varianzas. Las comparaciones múltiples entre grupos fueron realizadas utilizando la prueba de Tukey-Kramer. Los valores de $\alpha<0,05$ fueron considerados estadísticamente significativos. Los análisis fueron hechos usando el software JMP versión 7 de SAS Institute.

\section{RESULTADOS}

Los resultados del análisis histológico muestran que los animales a los que se les administró PPT una hora antes de ser sometidos al protocolo de hipoxia presentan, en todas las áreas estudiadas, menor daño que los animales que no fueron tratados con PPT (Tabla I). En el cerebro de los animales con- trol, las neuronas exhiben una morfología normal, i.e. membrana nuclear con bordes bien definidos, cromatina fina, núcleos evidentes y neurópilo homogéneo (Fig. 1a, d y g). Sin embargo, en los animales que no fueron tratados con PPT se presenta mayor cantidad de células dañadas en las cuales se observa un proceso de acidofilia gradual, evidenciada por el oscurecimiento de los núcleos y citoplasma, contracción del pericarion y pérdida de los límites de la membrana citoplasmática y nuclear con desorganización celular total (Fig. 1b, e y h). El daño que presenta el grupo de animales con tratamiento de PPT, una hora antes del evento hipóxico, está representado por la presencia de algunas células picnóticas alternadas con células normales (Fig. 1c, f y i)

Los datos de los grupos a los que se les administró PPT inmediatamente después de ser sometidos a hipoxia, no se muestran ya que el daño que se observa es similar al del grupo que no recibió PPT evidenciando que a nivel morfológico la administración de PPT bajo este esquema no protegió del daño inducido por la hipoxia.

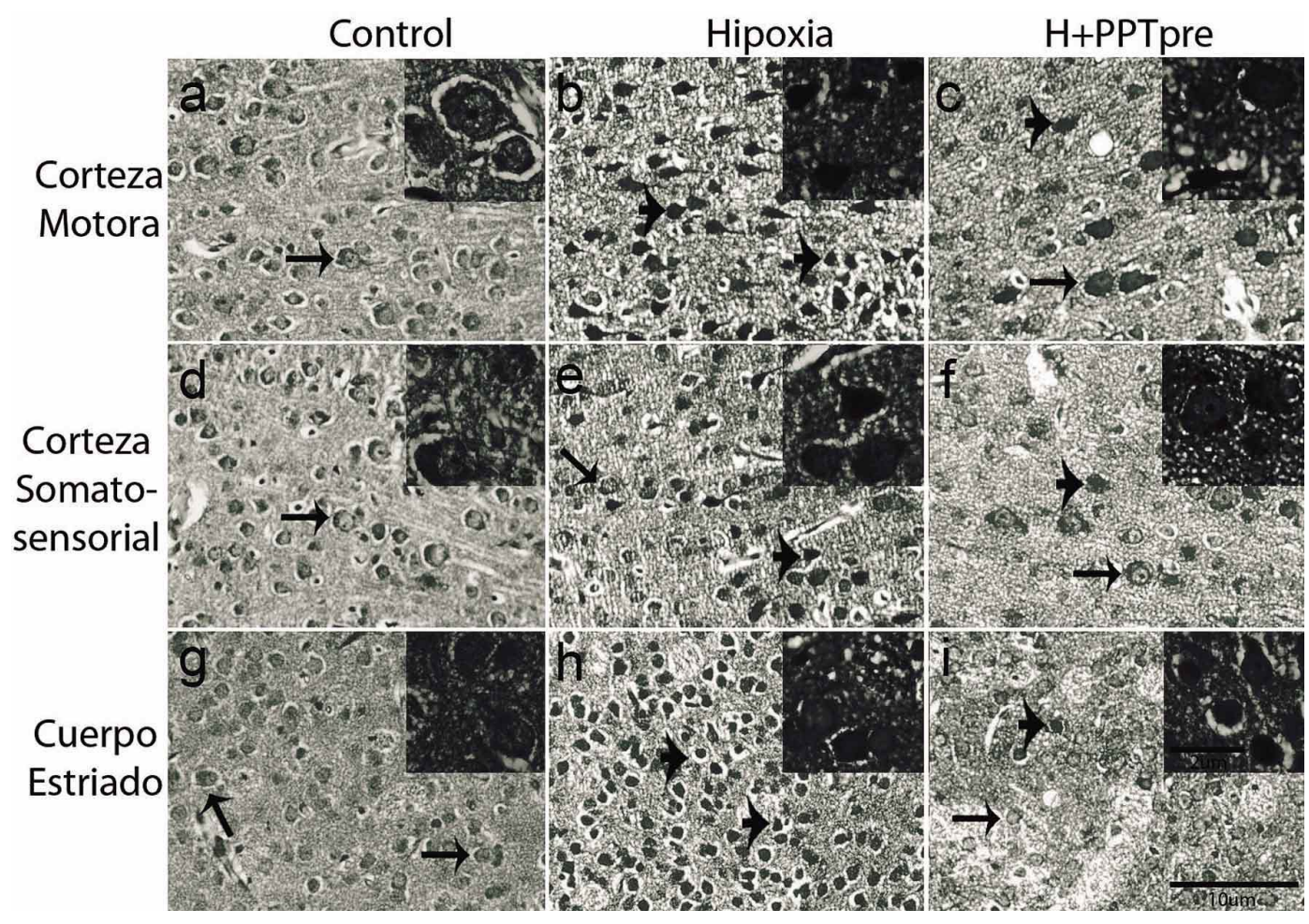

Fig. 1. Fotomicrografías que muestran 3 diferentes zonas del cerebro de los animales control (a, d, g), sometido a hipoxia (b, e, h) y con tratamiento de PPT una hora antes del evento hipóxico (c, f, i). Las células normales exhiben una morfología con membrana nuclear con bordes bien definidos, cromatina fina, núcleos evidentes y neurópilo homogéneo (flechas; ver recuadros). Las células dañadas presentan acidofilia gradual, evidenciada por el oscurecimiento de los núcleos y citoplasma, contracción del pericarion y pérdida de los límites de la membrana citoplasmática (cabeza de flecha; ver recuadros). Los animales que no fueron tratados con PPT presentaron gran cantidad de células dañadas, mientras que los animales tratados con PPT, una hora antes del evento hipóxico, muestran la presencia de algunas células picnóticas alternadas con células normales. 


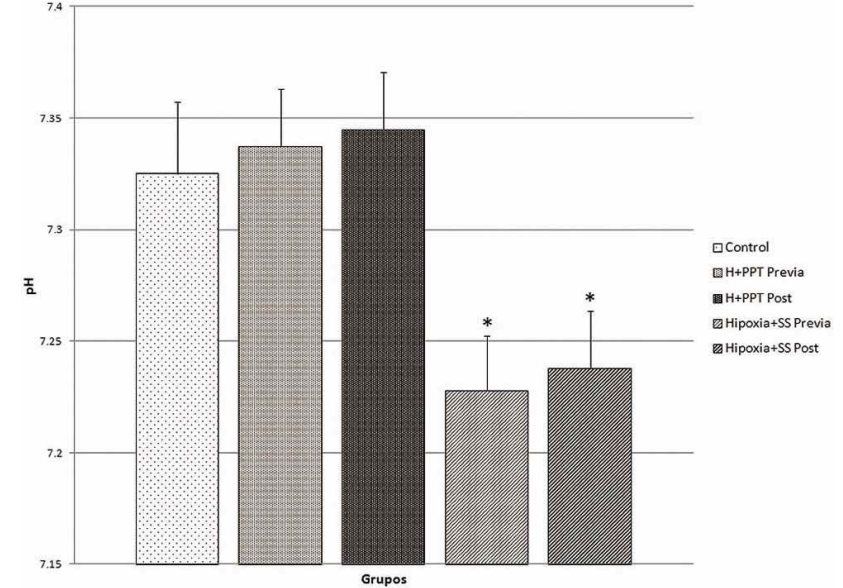

Fig. 2. Los animales que no recibieron tratamiento con PPT muestran valores significativamente menores de $\mathrm{pH}$, en tanto que en los tratados con PPT el pH es similar al del grupo control. Los datos presentan el promedio $\pm \mathrm{EE} ; * \mathrm{p}<0.05$.

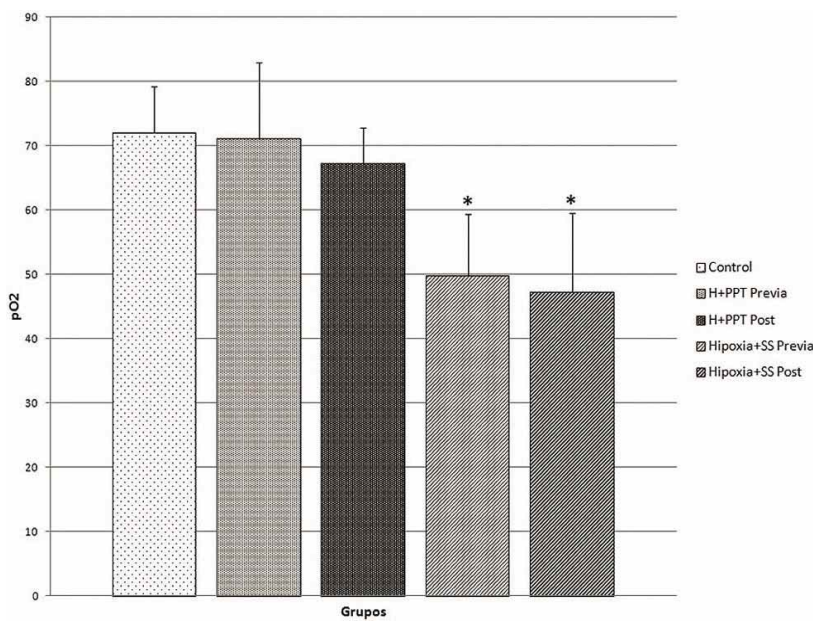

Fig. 3. Se aprecia una disminución significativa en la presión parcial de $\mathrm{O}_{2}$ en los animales sometidos a hipoxia en comparación con el grupo control y los que recibieron PPT. Los datos presentan el promedio $\pm \mathrm{EE} ; * \mathrm{p}<0.05$.

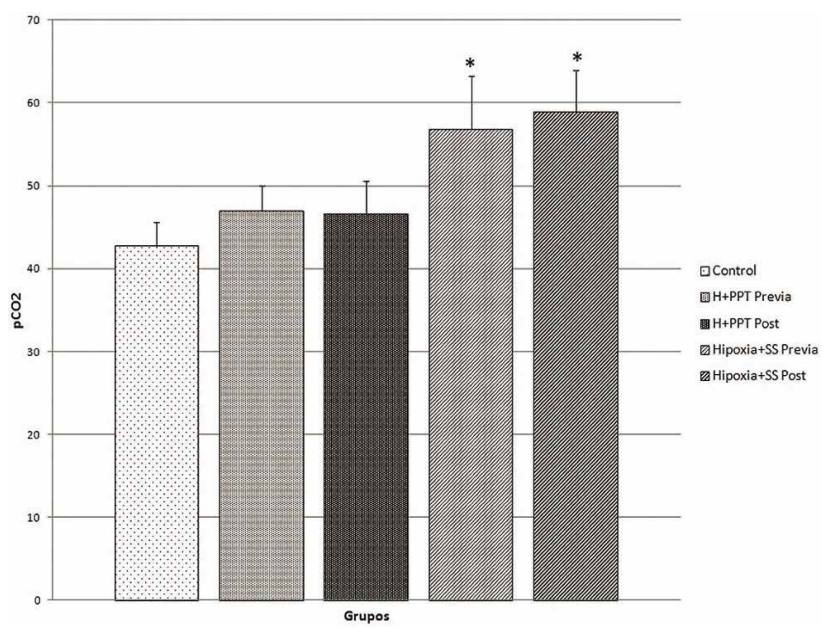

Los resultados del análisis gasométrico de las muestras sanguíneas de los grupos tratados con PPT una hora antes de la hipoxia y al término de esta, revela que todos los indicadores tienen valores semejantes a los del grupo control. Mientras que los animales que no recibieron tratamiento con PPT, muestran valores significativamente menores de $\mathrm{pH}$ y $\mathrm{PaO}_{2}$, y mayor concentración de $\mathrm{PaCO}_{2}$ con respecto del grupo que recibió PPT y el control (Figs. 2-4).

\section{DISCUSIÓN}

Los resultados de este estudio muestran una disminución del daño neuronal sólo cuando el PPT se administró de manera previa al evento hipóxico. En todas las áreas valoradas, los animales tratados con PPT antes de ser sometidos a hipoxia, presentaron menor daño neuronal que los animales con PPT posterior a la hipoxia o a los que no recibieron tratamiento. Estos datos sugieren que el PPT, cuando se administra previamente al insulto hipóxico, ejerce un efecto protector celular. Con la administración de PPT posterior a la hipoxia se observó un daño tisular similar a los animales que no recibieron PPT, esto indica que una vez que se ha iniciado el proceso de muerte celular prácticamente no existe posibilidad de revertirlo. Por otra parte, se observó que la administración de PPT ya sea previa o posterior al evento hipóxico normaliza los valores de $\mathrm{pH}, \mathrm{PaCO}_{2}$ y $\mathrm{PaO}_{2}$. Lo que concuerda con Lindenbaum \& Larrieu (1989) quienes observaron que la administración del PPT favorece la normalización del pH, la reserva alcalina y el consumo de oxígeno.

Como anteriormente se mencionó, la tiamina actúa como un cofactor de enzimas tales como la PDH y la $\alpha$ CGDH, cuya disminución puede conducir a una falla en la síntesis de ATP y contribuir a la muerte celular (Martin et al.). Al administrar el PPT, que es una molécula altamente energética, es probable que se provea esa energía extra para la producción de ATP que la célula necesita para reactivar los canales iónicos y los sistemas antioxidantes naturales del cerebro para sobrevivir a la hipoxia (Yager et al., 1992); permitiendo el funcionamiento normal de la célula o la activación de los mecanismos de detoxificación. Datos obtenidos en estudios in vitro sugieren que el PPT podría participar en: a) el reforzamiento del sistema antioxidante, funcionando como atrapador de radicales libres (Natera et al., 2011); b) la reducción de la acumulación excesiva de excitotoxinas ( $p$ ej. glutamato) y la reducción intracelular de la acumulación

Fig. 4. En los animales sometidos a hipoxia se observa incremento significativo de la presión parcial de $\mathrm{CO}_{2}$; los animales del grupo control y los que recibieron PPT muestran valores similares. Los datos presentan el promedio $\pm \mathrm{EE} ; * \mathrm{p}<0.05$. 
Tabla I. Semi-cuantificación del daño neuronal, encontrado en diferentes áreas cerebrales, de animales tratados y control.

\begin{tabular}{lccc}
\hline Área cerebral & Control & Hipoxia & H + PPTpre \\
\hline Corteza frontoparietal, área somatosensorial & $*$ & $* * *$ & $* *$ \\
Corteza frontoparietal, área motríz & - & $* * *$ & $* *$ \\
Corteza cingulada anterior & - & $* * *$ & $* *$ \\
Núcleo del limbo horizontal de la banda diagonal & - & $* * *$ & $* *$ \\
Núcleo premamilar, ventral y dorsal & - & $* * *$ & $* *$ \\
Núcleo hipotalámico posterior & - & $* * *$ & $* *$ \\
Área hipotalámica lateral & $*$ & $* * *$ & $* *$ \\
Cuerpo estriado o Putamen caudado & - & $* * *$ & $* *$ \\
Núcleo talámico ventroposterior & - & $* * *$ & $* *$ \\
Corteza estriada, área 18 & - & $* * *$ & $* *$ \\
Campo CA3 del cuerno del hipocampo & - & $* * *$ & $* *$ \\
Núcleo supraquiasmático preóptico & $*$ & $* *$ & $*$ \\
Corteza frontal & $*$ & $* *$ & $*$ \\
Corteza piriforme o corteza olfatoria primaria & $*$ & $* *$ & $*$ \\
\hline
\end{tabular}

Las células dañadas fueron semi-cuantificadas de la siguiente manera: ( - ) ausencia, (*) escaso, ( ** ) moderado, (***) abundante.

de calcio (Furukawa et al., 1997); y c) la inactivación de endonucleasas que producen peroxidación de lípidos y daño a la membrana (Dirnagl et al., 1999). Los resultados de esta investigación si bien muestran que hubo una reducción del daño celular a consecuencia de la administración previa del PPT, no son suficientes como para atribuir a algún mecanismo específico del potencial neuroprotector del PPT, por lo que futuros estudios son necesarios para conocer el mecanismo de acción de esta molécula ante el evento hipóxico.

Algunas investigaciones han mostrado que la tiamina tiene efectos benéficos en varios trastornos del cerebro de pacientes pediátricos, por ejemplo, en bebés de meses de edad con síndrome de West reduce los niveles de lactato y disminuye la frecuencia de los espasmos (Naito et al., 1999). En la encefalopatía de Wernicke, la administración de altas dosis de tiamina por vía intravenosa ha resultado en un tratamiento eficaz (Watanabe et al., 2009). Lo anterior indica que la suplementación con tiamina podría reducir, en alguna medida, el daño neuronal en humanos recién nacidos. Nuestros resultados muestran que el PPT tiene efecto sólo si es administrado antes del evento hipóxico, y aunque resulta complicado determinar con antelación si un organismo estará o no expuesto a un evento de este tipo en la etapa neonatal, resultaría interesante valorar el potencial del PPT como agente neuroprotector, en situaciones de alto riesgo, p.ej. en procedimientos de parto complicado, identificación de sufrimiento fetal, etc.

CONCLUSIONES. La administración de PPT, una hora antes de un insulto hipóxico, reduce el daño neuronal y estabiliza las constantes sanguíneas de $\mathrm{pH}, \mathrm{PaCO}_{2} \mathrm{y} \mathrm{PaO}_{2}$, en ratas neonatas. Estos hallazgos son congruentes con la hipótesis de que la pérdida de aporte energético, desencadena una serie de eventos que culminan con la muerte celular y que la preservación de la ruta metabólica que provee de energía a la célula puede reducir en alguna medida el daño neuronal.

AGRADECIMIENTOS. A los laboratorios Manuell por donar la Carzilasa (PPT), a la EBC. Lina Romero Guzmán Jefa del Laboratorio de Química Clínica del Instituto Nacional de Pediatría por su valiosa ayuda en la realización de los análisis de gasometría hemática, y al Dr. Bryan Phillips Farfán por la traducción del resumen.

VALENZUELA, P. A.; MARTÍNEZ, A. M. A.; JIMÉNEZ, B. M. A.; GRANADOS-ROJAS, L.; MENDOZA-TORREBLANCA, J. G. \& BARRAGÁN, M. M. G. Thiamine pyrophosphate reduces hypoxia-induced cellular damage in neonatal rat brain. Int. J. Morphol., 32(2):531-536, 2014.

SUMMARY: Hypoxic encephalopathy is a leading cause of disability and requires new therapeutic strategies. Thiamine pyrophosphate (TPP) is an essential cofactor of fundamental enzymes involved in glucose metabolism. TPP reduction may lead to ATP synthesis failure and cell death. The objective of this study was to determine if TPP administration can reduce cellular damage in a model of neonatal hypoxia in rats. Eleven day old animals were treated with TPP $(130 \mathrm{mg} / \mathrm{kg})$ as a single dose or with saline solution one hour before the hypoxia protocol or after ending the protocol. The brains were collected to evaluate cellular damage. Blood samples were also collected to evaluate arterial oxygen tension $\left(\mathrm{PaO}_{2}\right)$, carbon dioxide tension $\left(\mathrm{PaCO}_{2}\right)$ and acidity $(\mathrm{pH})$. The results showed that TPP administration previous to hypoxia induction reduces cellular damage and reestablishes arterial blood gases. These data indicate that TPP use reduces the damage induced by hypoxia in neonatal animals.

KEY WORDS: Thiamine pyrophosphate; Hypoxia; Neuronal damage; Arterial blood gas. 


\section{REFERENCIAS BIBLIOGRÁFICAS}

Delivoria-Papadopoulos, M. \& Mishra, O. P. Mechanisms of cerebral injury in perinatal asphyxia and strategies for prevention. J. Pediatr., 132(3 Pt. 2):S30-4, 1998.

Dirnagl, U.; Iadecola, C. \& Moskowitz, M. A. Pathobiology of ischaemic stroke: an integrated view. Trends Neurosci., 22(9):391-7, 1999.

Ferriero, D. M. Neonatal brain injury. N. Engl. J. Med., 351(19):1985-95, 2004.

Furukawa, K.; Fu, W.; Li, Y.; Witke, W.; Kwiatkowski, D. J. \& Mattson, M. P. The actin-severing protein gelsolin modulates calcium channel and NMDA receptor activities and vulnerability to excitotoxicity in hippocampal neurons. $J$. Neurosci., 17(21):8178-86, 1997.

Gunn, A. J.; Gunn, T. R.; de Haan. H. H.; Williams, C. E. \& Gluckman, P. D. Dramatic neuronal rescue with prolonged selective head cooling after ischemia in fetal lambs. J. Clin. Invest., 99(2):248-56, 1997.

Hass, R. H. Thiamine and the brain. Annu. Rev. Nutr., 8:483-515, 1988.

Langmeier, M.; Folbergrová, J.; Haugvicová, R. \& Riljak, V. Neuroprotective effect of (R, S)-4-phosphonophenylglycine against neuronal damage associated with homocysteic acidinduced seizures in immature rats. Prague Med. Rep., 107(4):421-32, 2006.

Lindenbaum, G. A.; Larrieu, A. J.; Carroll, S. F. \& Kapusnick, R. A. Effect of cocarboxylase in dogs subjected to experimental septic shock. Crit. Care Med., 17(10):1036-40, 1989.

Martin, P. R.; Singleton, C. K. \& Hiller-Sturmhöfel, S. The role of thiamine deficiency in alcoholic brain disease. Alcohol Res. Health, 27(2):134-42, 2003.

Naito, E.; Ito, M.; Yokota, I.; Saijo, T.; Chen, S.; Maehara, M. \& Kuroda, Y. Concomitant administration of sodium dichloroacetate and thiamine in west syndrome caused by thiamine-responsive pyruvate dehydrogenase complex deficiency. J. Neurol. Sci., 171(1):56-9, 1999.

Natera, J.; Massad, W. A. \& García, N. A. Vitamin B1 as a scavenger of reactive oxygen species photogenerated by vitamin B2. Photochem. Photobiol., 87(2):317-23, 2011.

Rice, J. E. 3rd.; Vannucci, R. C. \& Brierley, J. B. The influence of immaturity on hypoxic-ischemic brain damage in the rat. Ann. Neurol, 9(2):131-41, 1981.

Rozanov, V. A.; Abu Sali, I. I. \& Rozanov Ala. Neurometabolic effects and antihypoxic activity of a vitamin-coenzyme complex, including thiamine pyrophosphate, lipoate, 4phosphopantothenate, nicotinate and flavinadenine mononucleotide. Vopr. Med. Khim., 36(6):66-9, 1990.

Sameshima, H. \& Ikenoue, T. Hypoxic-ischemic neonatal encephalopathy: animal experiments for neuroprotective therapies. Stroke Res. Treat., 2013:659374, 2013.

Shin, B. H.; Choi, S. H.; Cho, E. Y.; Shin, M. J.; Hwang, K. C.; Cho, H. K.; Chung, J. H. \& Jang, Y. Thiamine attenuates hypoxia-induced cell death in cultured neonatal rat cardiomyocytes. Mol. Cells, 18(2):133-40, 2004.

Vannucci, R. C.; Connor, J. R.; Mauger, D. T.; Palmer, C.; Smith, M. B.; Towfighi, J. \& Vannucci, S. J. Rat model of perinatal hypoxic-ischemic brain damage. J. Neurosci. Res., 55(2):15863, 1999.

Vannucci, R. C.; Towfighi, J.; Brucklacher, R. M. \& Vannucci, S. $\mathrm{J}$. Effect of extreme hypercapnia on hypoxic-ischemic brain damage in the immature rat. Pediatr. Res., 49(6):799-803, 2001.

Watanabe, S.; Yamakura, S.; Hirano, K.; Okumura, Y. \& Aiba, H. Case of infantile autism with pediatric Wernicke's encephalopathy due to severe eating disorder. No To Hattatsu, 41(1):43-6, 2009.

Yager, J. Y.; Brucklacher, R. M. \& Vannucci, R. C. Cerebral energy metabolism during hypoxia-ischemia and early recovery in immature rats. Am. J. Physiol., 262(3 Pt. 2):H672-7, 1992.

Zehendner, C. M.; Luhmann, H. J. \& Yang, J. W. A simple and novel method to monitor breathing and heart rate in awake and urethane-anesthetized newborn rodents. PLos ONE, 8(5):e62628, 2013.

Dirección para Correspondencia:

Armando Valenzuela Peraza

Laboratorio de Neuroquímica

Instituto Nacional de Pediatría

Insurgentes Sur 3700-C, Col.

Insurgentes Cuicuilco, Del. Coyoacán

México D. F., 04530

MÉXICO

Email: avalenvuelap@pediatria.gob.mx valenzuela.peraza2013@hotmail.com

Recibido : 11-11-2013

Aceptado: 30-03-2014 